\title{
EL MUNICIPIO DE BARCELONA Y SU AYUNTAMIENTO
}

\section{II. - SERVICIOS MUNICIPALES.}

No es posible, en el breve espacio de un artículo, dar una idea exacta, y menos detallada, de todos y cada uno de ellos, sino sólo una impresión, más cuando la exposición del crecimiento material de la Ciudad, adoptado como tema principal, ha debido ocupar ya tanto espacio.

Vamos, pues, a limitamos a dar una idea elemental de los más importantes, clasificados en los siguientes apartados:
A) Vía Pública.
B) Transportes.
C) Abastos.
D) Cultura.
E) Higiene y Sanidad.
F) Beneficencia.

Al hacerlo así hemos seguido la distribución de trabajo entre las Tenencias de Alcaldía Delegadas que rigen las respectivas materias y presiden las Ponencias del Ayuntamiento en Pleno en su actual organización.

No olvidaremos, a pesar de lo somero del análisis, exponer sus deficiencias y señalar sus soluciones y proyectos, porque en este artículo, más que a dar cuenta de los servicios, se atiende a señalar los escollos que se han presentado, para que puedan evitarse en lo sucesivo y para que esta experiencia, aunque en lo elemental, pueda ser útil. Cada uno de esos servicios pudiera ser objeto de una monografía, por lo menos, con sólo recoger datos y proyectos desperdigados. No peco de indiscreto si digo que tales elementos los tiene el Ayuntamiento de Barcelona, a disposición de los estudiosos. Es 64 labor muy grata proporcionarlos frecuentemente a compañeros, tan- 
to más a Corporaciones hermanas, que así pueden tener al alcance de su mano la experiencia viva en cualquier materia.

\section{A) Los servicios de la vía pública.}

El ingente problema de urbanizar la Ciudad lleva aparejado el de dotar a ella y a sus vías públicas de los servicios indispensables para la vida y su reglamentación.

Estos servicios, anejos a los de urbanización, son de un. desarrollo y complejidad distintos, pues aquélla, después de llevarse a efecto, queda extática, en tanto que éstos han de ser constantemente vivos y cambiables y estar en continua actividad. Comprenden el alcantarillado, con el destino de las aguas residuales, pavimentos, limpieza de la vía pública y domiciliaria y suministro de luz y agua de carácter público.

1. Alcantarillado. - La ligera pendiente de la Ciudad al mar movió a llevar las aguas residuales a éste, con el consiguiente perjuicio para la salubridad y limpieza de la playa, que no pudo superarse a pesar de establecer la salida de los desagües algunos kilómetros dentro del mar, pues el oleaje ha hecho insuficientes tales medidas. La existencia de alguna zona urbana de nivel inferior al mar en el subsuelo es otro de los problemas fundamentales a resolver, pues el crecimiento rápido de la Ciudad obligó a construir la red de alcantarillado en toda ella, sin que diera tiempo a solventar tales problemas producidos en la parte inferior colectora, o sea en la desembocadura de la red en el mar. Proyectado el Ensanche en 1860, no se inició el estudio del alcantarillado hasta 1884, aprobăndose el proyecto general, al que tuvo que adaptarse lo ya construído cinco años después, o sea en 16 de junio de 1891, circunstancias que explican las deficiencias apuntadas, fruto de aquella improvisación, a que tantas veces nos hemos referido.

Para conseguir la solución de tales defectos, se impone la instalación de una estación elevadora y depuradora de las aguas residuales, que, emplazada en la zona cercana al mar, consiga ambas finalidades, para lo que el Ayuntamiento tiene dispuestos ya unos terrenos de su propiedad. Tal instalación se ha presupuestado en unos 40 millones de pesetas, y si bien la actual situación económica de la Ciudad y la especialidad de la maquinaria no permiten hoy realizarla, tendrá indudablemente que abordarse en plazo breve.

El sistema del alcantarillado, adoptado a propuesta del Ingeniero de Caminos, autor del proyecto, don Pedro García Faria, fué el unita- 65 
rio por el que se acarrean las aguas residuales y las de lluvia por las mismas galerías, todas ellas visitables y dispuestas con cunetas y carriles para transportar las vagonetas de limpieza. Una serie de depósitos de agua con descarga automática, evita los sedimentos. En alguna zona poco poblada se adopta el sistema separativo, por secciones de poco diámetro no visitables. Su conservación y limpieza, aunque municipalizada, como es lógico, está contratada mediante cuadros de precios unitarios para cada labor especial, evitando así el mantenimiento del cuantioso personal que su sostenimiento impone. Estas son las características técnicas más destacables, y a ellas podemos añadir un dato estadístico impresionante: la extensión de la red alcanza hoy a 519 kilómetros lineales.

2. Pavimentos-Diversas son las clases de pavimentos empleados actualmente en Barcelona, para que cada uno de ellos responda a las necesidades derivadas de la distinta intensidad del tráficc, pendiente y demás circunstancias especiales de las vías a que se destinan.

Así, desde el simple afirmado de tierra y cascote para pasens y jardines, a los pavimentos de piedras duras sobre blindaje de hormigón en vías de tránsito pesado, se emplean el macadam con riegos de alquitrán, betún o asfalto, de mayor o menor penetración en su masa; el pavimento asfáltico en polvo comprimido, loseta o fundido, sobre cimiento de hormigón; el adoquinado con piedras más estrechas que las empleadas antiguamente y colocadas en hileras perpendiculares a la dirección del tránsito; el pequeño adoquín mosáico, etcétera, etc.; se ensayan, además, continuamente los principales pavímentos modernos.

Como piedras blandas, se utilizan la arenisca de Montjuich y las calizas; como duras, principalmente los granitos, pórfidos y el basalto.

La madera, en tarugos sobre hormigón, no ha dado en Barcelona resultados satisfactorios.

En las aceras ya no se emplean las antiguas losas de arenisca de Montjuich, a causa de su desigual desgaste. Se admiten las de piedras homogéneas, pero se pavimentan principalmente con baldosillas cuadradas de hormigón, de 20 centímetros de lado, chapadas de cemento con dibujos refundidos, asentadas sobre hormigón.

Con objeto de no remover constantemente el pavimento de las calles, facilitando el empleo de estructuras monolíticas con los materiales modernos, ventajosos por su acomodación al tránsito, falta 66 de sonoridad y facilidad que prestan para el empleo de las máquinas 
de limpieza modernas, se aprobaron en 1915 los perfiles transversales del subsuelo de las calles, en los que se fijan las distancias a las fachadas y la profundidad a que deben situarse debajo de las aceras, en función de la anchura de éstas, las distintas conducciones de distribución del agua, gas, electricidad, teléfonos, etc., con doble distrihución de estos fluídos en cada calle, excepto en las estrechas. Para idéntica finalidad se han empleado otros procedimientos. La superficie total de pavimentos construídos es de 8.796.510 metros cuadrados.

3. Limpieza de la vía pública.-Se presta este servicio, así como el de recogida de las basuras domiciliarias, por una empresa particular, según contrato aprobado por el Ayuntamiento, en Junta de Vocales Asociados, en 17 de mayo de 1915, objeto de distintas modificaciones y prórrogas posteriores.

La pavimentación y limpieza de las calles se practicaba antes por brigadas municipales, en las que se ingresaba con carácter provisional, mas las dificultades que luego se presentaban para eliminar el personal inadecuado obligaron a prescindir de tales nombramientos, pasándose a un contratista tales brigadas, de las que se reservó el Ayuntamiento algunos empleados que habían probado su aptitud como Inspectores, Capataces, etc.

4. Luz y agua.-Ambos servicios se encuentran en manos de Compañías privadas, tanto en lo que se refiere al suministro público como al particular. Cierto que, desde la vigencia del Estatuto Municipal, el Ayuntamiento viene participando en el producto bruto de las Empresas, de conformidad con lo dispuesto en el artículo 378 de dicho Estatuto, con la ventaja de conocer, por tanto, sus gastos de expltación y balances, pero sin control ni intervención alguna aun cuando ello le proporciona interesantes datos para el estudio de su posible municipalización.

En cuanto a los de iluminación, tal situación, impuesta por las circunstancias a que repetidamente se ha venido haciendo referencia, había llegado hasta el punto de que, excepto los candelabros, las instalaciones no eran de propiedad municipal. Hoy día, si bien la red de distribución no es aún propiedad del Municipio, está prevista la forma en que pasará a serlo en lo sucesivo. El vigente contrato de suministro de flúido eléctrico para el alumbrado público prevé, además, la instalación de contadores, ya que hasta ahora se abonaba un tanto alzado por $\mathrm{Kw}$. hora de lámpara instalada, lo que, por las dificultades de la diaria inspección de los faroles fuera de servicio, encarece el suministro. 
La iluminación de Barcelona es mixta, parte eléctrica y parte por gas, estando instalada en la zona de Ensanche la primera, y en la de Interior, incluídos los pueblos agregados, la segunda; en algunos puntos, como la Plaza de Cataluña, por ejemplo, el Paseo de Gracia y la parte central de la Avenida de José Antonio, coexisten ambos sistemas.

Son públicas las ventajas que representa la dualidad de sistema, ya que si, por cualquier circunstancia, como ocurre actualmente por falta de carbón, se dificulta o imposibilita el suministro de gas, no queda a oscuras la integridad de la Ciudad, sino tan sólo algunos sectores de la misma, en tanto que los iluminados eléctricamente conservan el servicio, o viceversa, como ocurrio durante la dominación roja, cuando, ocupadas las centrales eléctricas por el Ejército Nacional, casi un año antes de la liberación de Barcelona, dejaron de suministrar el flúido, quedando en cambio en servicio la iluminación por gas, que hubiera podido continuarse si el oscurecimiento de la Ciudad no hubiera aconsejado a los rojos su apagado.

El número de faroles de gas es de 6.035, y los de electricidad instalados definitivamente de 6.785, quedando aun instaladas provisionalmente 11.240 luces, lo que da un total de 24.060 , que suponiendo una distancia promedia de 20 metros, da un total de extensión de las línea de alumbrado público de más de 480 kilómetros.

En cuanto a los servicios de aguas, bastará decir que en el año 1912, y ante la necesidad de una reforma y de aumento de caudal se iniciaron los trámites para la municipalización de los mismos, sin que hasta la fecha se haya resuelto tal problema. El estudio llevado a cabo en tal fecha fué publicado en un volumen en cuarto de 312 páginas, y no contiene la integridad del expediente. Como puede comprenderse fácilmente, desde tal fecha hasta hoy, dado el crecimiento de la Ciudad, el problema presenta una agudización que lo coloca en primer plano; mas cuando el caudal de aguas de que se nutre la Compañía explotadora del servicio aparece contaminado por transcurrir por las zonas salineros de Cardona y Suria, y por utilizar las minas de potasa de ella tales aguas para la limpieza del mineral, lo que ha obligado a estudiar la construcción de un canal colector de las aguas residuales de las mismas, que defienda en lo posible la pureza de las aguas, sin que, empero, pueda estimarse ello como una solución definitiva, apesar del coste de $\mathbf{1 0 . 0 0 0 . 0 0 0}$ de pesetas, en que ha sido proyectada la obra.

El Ayuntamiento posee, por su parte, una captación en el pueblo 68 limítrofe de Moncada, cuyas aguas dieron lugar, en el año 1914 a 
una epidemia tifoidea, y a ráŕ de la cual fué instalada una estación depuradora y elevadora, con excelentes resultados, pero con un coste de instalación y de explotación que resulta exagerado por lo reducido del caudal.

Entre los caudales de que dispone la Compañía de Aguas, otros captados por particulares en la propia Ciudad y los del Ayuntamiento, ascedentes en junto a 371.000 metros cúbicos diarios, la cantidad de agua de que se dispone por habitante es tan sólo de 28 litros, a todas luces insuficiente para asegurar el mínimo indispensable a su higiene.

Como puede verse, los servicios incluídos en este último apartado de los que comprenden el presente grupo aparecen en situación de franca inferioridad respecto de los anteriores, y su solución es imperiosa y vital para la Ciudad, y para su Ayuntamiento; por la primera, para conseguir una mejora en el servicio, y para éste por el importante volumen de ingresos que puede representarle la municipalización de tal clase de servicios. Ahora bien, la ejecución del plan de municipalización obligará a la inversión de decenas de millones de pesetas, de los que hasta hoy no ha podido disponer el Ayuntamiento, tan mal parado en su Hacienda.

Cuando las operaciones de saneamiento de su situación financiera, a que deberemos referirnos en otro artículo, hayan dado la plenitud de su rendimiento, es indudable que podrá pensar formalmente en tal clase de negocios con completa garantía de éxito.

\section{B) Los servicios de transportes.}

Desde la primera concesión de líneas de tranvías por tracción de sangre hasta los modernos filobuses puestos en servicio hace más de un año, el Ayuntamiento no se puede decir, no ya que dirigiera, sino ni siquiera que presidiera, los transportes urbanos. La legislación anterior al Estatuto Municipal, que hacía depender las concesiones exclusivamente del Ministerio de Obras Públicas, restaba a la Corporación, no sólo iniciativas, sino toda autoridad en la materia. El precepto del Estatuto Municipal, declarando de competencia de los Ayuntamientos cuanto hace referencia a transportes urbanos, no mejoró de momento en gran cosa los términos del problema, pues el Ministerio de Fomento o de Obras Públicas, a instancias de las Compañías, había ido concediendo las líneas que aquéllas, en previsión del crecimiento de la ciudad, iban solicitando; la competencia municipal se encontraba, pues, limitada por tales situaciones 
de derecho, que era imposible económicamente expropiar. Por otra parte, la discusión constante sobre el tema de la competencia de los tranvías tanto los de superficie como.los subterráneos y los interurbanos, por parte del propio Ministerio, dificultaba entonces y continúa hoy enervando la iniciativa municipal en forma tal, que, a pesar de llevar casi veinte años de vigencia tal precepto, nada se ha podido hacer prácticamente en la materia.

Expuestas estas líneas generales de la situación, es preciso sentar algunos antecedentes en relación a la Ciudad de Barcelona. En ella coexistían seis Compañías de Tranvías, con dos anchos distintos; dos de Autobuses, con diversas concesiones municipales entremezcladas una de ellas en sus intereses con las propias Compañías de Tranvías; el Gran Metro de Barcelona, S. A., con su línea subterránea Lesseps-Puerto; el Ferrocarril Metropolitano de Barcelona, S. A. (Transversal), y el Ferrocarril de Sarriá a Barcelona.

Las primeras, o sea, las de Tranvías, tenían de hecho un monopolio del servicio por la superficie de la Ciudad, ya que la competencia de los autobuses independientes era insignificante.

Por su parte, el Ayuntamento tenía el control del Ferrocarril Metropolitano Transversal, y una esperanza próxima de explotación del Ferrocarril de Sarriá a Barcelona, en virtud de'la reversión acordada a su favor.

Sobre estos dos últimos vamos a hacer una somera explicación. El Metro Transversal, constituído en Sociedad Anónima, en el año 1920 para atravesar la Ciudad de S. O. a N. O., fué concedido por el Ministerio de Obras Públicas con carácter de ferrocarril secundario y sin subvención ni garantía de interés. Su capital desembolsado de 15.000 .000 de pesetas fué suscrito en cuanto a 5.000.000, por la Corporación Municipal. Ejecutado en parte, y ante dificultades económicas difíciles de superar, se estableció un convenio entre el propio Ferrocarril Metropolitano, el Ayuntamiento y la Compañía de los Caminos de Hierro del Norte de España en el año 1928 para la terminación del ramal Plaza de Cataluña a Estación del Norte, con cuatro vías, para que los trenes-tranvías del Norte pudieran rendir viaje en el centro de la Ciudad; el Ayuntamiento avalaba una emisión de obligaciones de $\mathbf{9 . 0 0 0 . 0 0 0 ~ d e ~ p e s e t a s , ~ a ~ c a m b i o ~ d e ~ l o ~ c u a l ~ o b - ~}$ tenía la mayoría en la Junta General y en el Consejo de Administración, y el Estado renunciaba a los derechos de reversión de la línea, cediéndosela al Ayuntamiento al fin de la concesión. Es el único Metropolitano de España municipalizado, no obstante lo cual se ha 70 pretendido incluirlo en la Ley de Rescate de los Ferrocarriles, para 
integrarlo en la Red Nacional de Ferrocarriles Españoles, si bien el expediente, aun en trámites, ha sido dictaminado favorablemente por el Consejo de Estado, el cual, haciendo por primera vez aplicación de la disposición antes citada del Estatuto Municipal, reconoce ser de competencia municipal cuanto se refiere a transportes urbanos (1).

Este primer paso de intervención municipal en materia de transportes urbanos tuvo su continuación en el decreto de 26 de diciembre de 1924, por el que se autorizó al Ayuntamiento de Barcelona para transformar en subterráneo el Ferrocarril de Sarriá a Barcelona, suprimiendo su trazado de superficie por la calle de Balmes y por la Riera de San Gervasio; su concesión primera a vapor había sido transformada más tarde, por el crecimiento de la Ciudad, en eléctrico, con peligro enormes, pues las grandes unidades, aparte dificultar el tráfico, daban lugar a constantes accidentes.

Por tal decreto, a cambio del esfuerzo económico que representaba el que la Corporación Municipal transformara en subterráneo el Ferrocarril, se le concedía el derecho a la reversión al término de la concesión en el año 1955. Tenía, pues, el Ayuntamiento, una obra ejecutada por valor de más de 30.000 .000 de pesetas, sin rendimiento durante más de veinticinco años, pero que le aseguraba una intervención real y efectiva en materia de transportes urbanos, en el porvenir, lo que, unido a la posesión del control del Ferrocarril Metropolitano Transversal, le colocaba en situación de poder abordar integramente la materia de transportes urbanos, cuando comenzaran. a revertir a su favor las primeras líneas de tranvías, concedidas en la segunda mitad del siglo pasado.

Ello ocurrió a raíz de la liberación de Barcelona, pues había ya revertido la línea número 1, Sans-Ramblas, y vencía a los pocos meses la línea Barcelona-San Andrés. Se unió a tal coyuntura favorable el despojo y el destrozo que los comités rojos de incautación habían hecho en el material móvil de autobuses y tranvías y la necesidad de reposición de carriles y cables, que tres años de continuo abandono, sin nuevas instalaciones ni reparaciones, hacía urgente.

No es dable en los términos de este artículo, ni por quien lo redacta, entrar a examinar y a criticar el convenio de explotación firmado entre el Ayuntamiento y Tranvías de Barcelona, S. A., en nombre y representación de todas las Compañías de Tranvíasi que discurrían por la superficie, y en nombre también de la Compañía General de Autobuses. Baste con indicar aquí sus características. Por

(1) Véase el articulo publicado sobre este caso en el núm. 19-20 de la Revista de Gobierno y Administración Local (1941). 
el contrato no se establece monopolio, pero sí se règularizan las líneas existentes y se prevé la aportación por el Ayuntamiento de otras nuevas, en número y calidad suficiente para atender a las necesidades urbanas en un futuro próximo y la transformación de las líneas de vía estrecha por filobuses o autobuses térmicos. El Ayuntamiento tiene una representación minoritaria y otra técnica en el Consejo de Administración de la Compañía, principalmente para cuanto hace referencia a la explotación; el Alcalde es el Presidente nato de la Compañía y del Consejo, y tiene facultades de veto para determinados acuerdos de ambos; el Ayuntamiento percibe en concepto de imposición municipal el 4 por 100 de la recaudación bruta, y en aquellas líneas revertidas ya, o que vayan revirtiendo en lo sucesiva, así como en las de nueva explotación, otro 4 por 100 más, o sea el 8 por 100 en conjunto; se unifican los plazos de reversión estableciéndose razonadamente por líneas y recorridos, destruyéndose así la tela de araña que habían edificado las Compañías para dificultar este tránsito de hoy; y se les obliga a la reposición de las instalaciones y del material, sin tener en cuenta el plazo que quede para la reversión, así como a invertir en pavimentos la suma de 15.000.000 de pesetas en aquellas vías en que se proyecta el establecimiento de filobuses o de autobuses térmicos.

Fruto de este convenio, que fué publicado íntegramente en la Memoria "La obra del Ayuntamiento durante los años 1939-40", en donde puede verse fué la puesta en servicio del filobús Marqués del Duero-San Andrés, en sustitución de la línea de autobuses térmicos del propio recorrido, y del tranvía de la Plaza Urquinaona-San Andrés, que, revertido ya al Ayuntamiento, se encontraba sin posibilidad de explotación por el estado de sus vías. y por la insuficiencia de sus coches anticuados. Este ensayo, que ha sido un verdadero éxito, confirma que; si las circunstancias hubieran sido las normales, hubieran podido llevarse a cabo, en el plazo previsto en el convenio, la transformación total de los servicios en la forma que en él se indica, o sea suprimiendo todas las líneas de vía estrecha, con sus coches pequeños, y sustituyéndolos por filobuses en una parte y en otra por autobuses térmicos, a cuyos resultados deberá llegarse forzosamente tan pronto como el ambiente de guerra cese y pueda disponerse del material preciso para ello.

El convenio reseñado ha de tener indudable resonancia cuando, resuelto favorablemente el expediente de rescate del Ferrocarril Metropolitano de Barcelona, S. A. (Transversal), vuelva su explotación 72 a la empresa, y, por tanto, al Ayuntamiento, al que se aproxima la 
reversión del Ferrocarril de Sarriá a Barcelona, en cuyo momento, estos primeros pasos de ingerencia, competencia y autoridad del Ayuntamiento en materia de transportes urbanos podrán consolidarse plenamente y tener total efecto, poniendo en sus manos íntegramente los mismos.

Los tres pasos preliminares para conseguir la municipalización están ya dados (mayoría en el Metropolitano Transversal, reversión del Ferrocarril de Sarriá a Barcelona y convenio con Tranvías), faltando tan sólo el último, en el que puede ya pensarse con la plena seguridad de un éxito rotundo.

Recordemos aquí que al construirse la vía Layetana lo fueron también unos túneles municipales que utiliza en parte el Gran Metro, y que por tanto en esta explotación colabora también èl Ayuntamiento, y añadamos ahora que en la parte superior de la calle de Balmes, desde la plaza de Gala Placidea, en donde está enclavada la Estación de Gracia, del Ferrocarril de Sarriá a Barcelona. hasta la avenida del Tibidabo, construyó. asimismo el Ayuntamiento un túnel para servicio ferroviario, y que por tanto, y estando establecido el enlace con el Ferrocarril de Sarriá a Barcelona, un nuevo ramal de servicio subterráneo está en sus manos con lo que queda reseñado todo el trabajo de preparación realizado en veinte años en la materia y que augura una fácil y próxima solución integral de la materia de transportes dentro de lo que para la vida de la Ciudad son unos años.

\section{C) Ios servicios de abastos.}

La tendencia de la legislación actual, que niega competencia a los Ayuntamientos de las capitales de provincia, en beneficio de los serviciós estatales en su organización provincial, ha estado a punto de producir un enorme colapso en varios ramos, entre los que el más destacado es el de Abastos. Tan solo la comprensión de las personas que los han regido y el tacto de las Administraciones municipales han podido evitarlo. No es un secreto para nadie decir que hoy el Ayuntamiento de Barcelona no tiene responsabilidad alguna en la materia y que tiene su propia organización al servicio del Estado. $\mathrm{Ni}$ los servicios-de padrón de habitantes, a efectos de racionamiento, en manos del Gobierno Civil; ni el Matadero ni los Mercados, están bajo su responsabilidad directa; pero ello no impide que la colaboración sea leal, amplia y eficaz.

Principalmente destacan los servicios de estadística de precios y de consumos, que, llevados con toda escrupulosidad por el Negocia- 73 
do de Estadística de la Sección de Abastos, facilitan elementos de juicio de enorme interés a los servicios provinciales, como asimismo el registro obligatorio establecido en el Negociado de Mercados y Comercios, de todos los establecimientos de comer, beber y arder, facilita a aquéllos elementos preciosos para su trabajo. Ni que decir tiene que la inspección sanitaria municipal, ejercida por el Cuerpo de Veterinaria, el Laboratorio Municipal y los servicios de inspección administrativa, colaboran con toda intensidad en tal obra.

El Ayuntamiento cuenta con 21 mercados, que representan un total de 8.607 puestos de venta en conjunto.

Además se encuentran en construcción dos nuevos mercados, uno sustituyendo el antiguo del pueblo agregado de Horta, que, situado en la antigua plaza, ha sido trasladado a un solar próximo, para ser instalado con toda modernidad, y otro en la barriada de la Sagrada Familia, en donde, utilizando un edificio apropiado a tal fin, se está procediendo a la instalación de sus puestos de venta y servicios complementarios.

En cuanto al Matadero, que si bien tiene una superficie de 58.892,25 metros cuadrados, equivalente a cuatro manzanas del Ensanche, no tiene toda la modernidad precisa para los servicios actuales, es otro de los problemas que tiene planteados la Ciudad, por cuanto su emplazamiento, mal escogido, lejos de las estaciones ferroviarias que lo abastecen, impone su traslado. Traslado que dada la capacidad del consumo, obliga a un madurado estudio, pues para la regularización del mercado sería altamente conveniente dispusiera de pastos que evitaran las continuas especulaciones en épocas normales, cuando, por algunas circunstancias o dificultad de transporte. se producen irregularidades en el abastecimiento del mercado de ganado.

Sería interesante reseñar cifras de consumo de la Ciudad, pero para ello nos remitimos nuevamente a la Memoria publicada por el Ayuntamiento de los años 1939-40, en la que constan tales datos por medio de gráficos y cuadros estadísticos, en los que resaltan la importancia y volumen de este ramo.

\section{D) Los servicios de Cultura.}

Se examinarán en este apartado cuatro grupos, constituídos por la Instrucción Primaria, las Escuelas de Artes y Oficios, los Archivos y Museos, y otras instituciones culturales, en cuyo conjunto se pueden considerar agrupados los que, bajo la competencia municipal,

74 están establecidos en la actualidad. 
1.' Instrucción Primaria.-Para facilitar locales a las Escuelas Nacionales, ha venido construyendo el Ayuntamiento Grupos escoiares, hasta un número de 36 , capaces para 22.000 alumnos, satisfaciendo además alquileres de distintos locales, para continuar las Escuelas unitarias en tanto se completa la edificación de Grupos escolares; ascienden aquéllas a 120 , con una capacidad de 8.000 alumnos, con cuyo conjunto se atiende tan solo la insuficiente cifra de 30.000 alumnos. Eilo representa una aportación a los servicios del Estado de 1.270.000 pesetas, por equivalencia de casa-habitación de los Maestros nacionales, que unida a los gastos de conservación y de alquileres de los edificios antes referidos, da un total de 2.363.100 pesetas, cifra de respetable importancia difícil de superar.

La materia es realmente inagotable, pues ningún nuevo esfuerzo, dentro de las posibilidades económicas en que se ha mantenido el Ayutamiento, es suficiente a absorber la integridad de la población escolar, cada día en aumento; ni siquiera en la época de la República, con la incautación de los tres enormes Colegios de los PP. Jesuítas, ni en la época roja, con la ocupación de los que no fueron pasto de las llamas, de las restantes comunidades religiosas, llegó a absorberse la población escolar.

Esta se cifra en 60.000 , por lo que, de acuerdo con las cifras dadas anteriormente, existe un déficit de 30.000 . plazas, que represen$\tan 600$ Maestros más, sobre los 700 y pico que actualmente prestan servicio, y la construcción de 30 Grupos escolares, de una capacidad media como los actuales. Para llegar a cubrir este déficit, antes de que el crecimiento de la Ciudad lo haga mayor, tanto el Estado como el Ayuntamiento, deberán efectuar un enorme esfuerzo. El Ayuntamiento, por su parte, en presupuesto extraordinario de obras recientemente ha aprobado, ha consignado la cantidad de $\mathbf{5 . 0 0 0 . 0 0 0}$ de pesetas para la construcción de cinco Grupos escolares, para los que cuenta ya con solares en los lugares apropiados de la Ciudad donde la densidad escolar desatendida es mayor. Véase cuánto hará falta todavía, y cómo el crecimiento de la Ciudad sigue imponiéndose a las posibilidades municipales.

La colaboración con el Estado en esta materia de instrucción primaria se presta, además, a través de Escuelas exclusiva y típicamente municipales, entre las que se cuentan cinco parvularios, cuatro Grupos al aire libre en los alrededores de la Ciudad, con cantina, y cuatro Escuelas más de deficientes, de las siguientes especialidades: sordomudos, atrasados, ciegos y deficientes. 
2.9 Escuelas de Artes y Oficios.-El Ayuntamiento mantiene por su cuenta, y sin perjuicio de la colaboración que presta con 14 cátedras de formación doméstica de la mujer en distintos Grupos escolares a cargo del Estado, cuatro Escuelas de tal especialidad con - su personal y material ciocente en las barriadas obreras más necesitadas.

Asimismo, en los Distritos $5 .^{\circ}, 6 .^{\circ}$ y $8 .^{\circ}$, sostiene sendas Escuelas complementarias de Artes y Oficios, en armonía y bajo la inspección técnica de la Junta Local del Instituto de Formación Profesional, con cuyos gastos de organización y sostenimiento cumple con notables creces las obligaciones que le corresponden en tal materia.

Completa este apartado la Escuela de Labores y Oficios de la Mujer,"en donde con toda amplitud se prepara a las niñas y a las adultas para las labores y trabajos domésticos de la confección, con sus especialidades de encajes y bordados, de costura y planchado, e incluso de dibujo de modelos y de historia del Arte. Esta Escuela tiene anejas varias de Corte y Confección.

3..$^{\circ} \quad$ Museos.-La organización actual de los Museos de la Ciudad, colocados bajo el alto Patronato del Estado, a través de una Junta creada por el Ministerio de Educación Nacional, ha dividido la labor que en la materia realizaban antes en conjunto el Ayuntamiento la Diputación, quedando a cargo del Ayuntamiento las siguiences mo tituciones: el Archivo Histórico de la Ciudad, con sus anejos el Museo de la Ciudad y el Museo de Artes e Industrias Populares, instalado en el Pueblo Español; el Museo de Cataluña, en el que fué $\mathrm{Pa}$ lacio Nacional de Montjuich, y el de Arte Moderno, en el antiguo parque de la Ciudadela, destinado también en parte a exposiciones especiales y periódicas, tales como la Exposición Nacional de Bellas Artes, recientemente celebrada; la de Arquitectura Alemana y la de la Federación de la Vivienda y Urbanismo.

Sin entrar en los detalles de las colecciones que albergan y de su organización y. del coste de su mantenimiento, digamos, para concluir este apartado, que el Museo Arqueológico y el Marítimo, a cargo de la Diputación, están instalados en edificios municipales, sitos, el uno, en el Parque de Montjuich, y el otro. en las Reales Atarazanas, y que la Biblioteca Central de la Diputación y el Instituto de Estudios Mediterráneos se encuentran asimismo albergados en el antiguo edificio del Hospital de la Santa Cruz, propiedad también de la Corporación.municipal.

La restauración de éste y de las Reales Atarazanas, así como 76 del antiguo Salón del Tinell y de la Casa Padellás en la plaza del Rey, 
donde se está instalando el Museo de Historia de la Ciudad, es un ejemplo vivo de cómo las instituciones de cultura del Ayuntamiento hacen revivir el cariño y el afecto a la Ciudad vieja y a los edificios históricos y artísticos que encierra, destinándolos a fines de alta cultura, que han de darles mayor popularidad e inmortalidad aún que aquélla que alcanzaron ya por su tradición histórica.

4. Otras instituciones.-La Escuela Municipal de Música, de reconocida fama en la Ciudad y fuera de ella, es únïca en su clase, tanto que por reciente disposición del Ministerio de Educación Nacional le ha sido concedido el rango de Conservatorio oficial, a pesar de ser en su organización, en sus servicios y en lo económico, estrictamente municipal. Puede decirse que es secuela de ella la Banda Municipal de Barcelona, cuyos conciertos gozan de gran popularidad y cuyos éxitos en el extranjero, en épocas de paz, tanto nombre y fama dieron a la Ciudad.

El Instituto de Ciencias Naturales es otra organización pura y exclusivamente municipal, teniendo a su cuidado la colección zool6gica, el aquarium y los Museos de Historia Natural, tanto de flora y fauna, como el minerológico y de insectos, siendo sus colecciones casi las únicas que se han salvado de la barbarie roja durante la revolución, por lo que su número de visitantes, principalmente de Colegios destinados a la segunda enseñanza, ha aumentado en número extraordinario en los últimos años.

$\mathrm{Y}$ otra demostración cultural son las ferias periódicas de muestras, que aún cuando quieren materializarse y presentarse en un aspecto solamente económico, de protección a la Industria y al Comercio de la Ciudad y de su provincia, son en definitiva el máximo exponente de su cultura. De nada servirían estas exhibiciones periódicas si su fin sólo fuera esta parte externa y visible de las transacciones comerciales. Su finalidad es más elevada, porque aparte acercar a Barcelona las restantes ciudades de España y sus habitantes y crear el estímulo entre unas y otros, tienen la virtud de producir en el interior este mismo estímulo. de superación, no sólo de unas casas comerciales sobre otras y de los ramos de la industria entre sí, sino de los propios individuos, con lo que se consigue el fin de alta cultura, que clasifica dichas ferias en este apartado.

La últimamente celebrada obligó al Ayuntamiento a un dispendio de 5.000.000 de pesetas para la reconstrucción de los edificios y parte de los jardines del Parque de Montjuich destinados a tal finalidad, y produjo un rendimiento, por la participación que la organización de la Feria dió a la Corporación municipal, de muy cerca del 77 
millón de pesetas; cifras que aseguran que tal esfuerzo económico ha de ser superado en muy pocos años, si no se considera ya saldado con los beneficios de orden cultural y comercial que la Feria, en el primer año de su exhibición después de la guerra de liberación, ha producido al conjunto de la Ciudad y a muchos de sus habitantes.

\section{E) Los servicios de Higiene y Sanidad.}

Para el examen y presentación de los de tal naturaleza, los consideraremos agrupados en tres apartados, o sea Higiene, Sanidad y Cementerios.

1. Higiene.-El Ayuntamiento cumple sus obligaciones higiénicas a través de tres organizaciones que se consideran como indispensables y mínimas, pero que pocos Ayuntamientos de España tienen establecidas con completa independencia unas de otras. Estas son el Laboratorio Municipal, el Instituto Municipal de Higiene y el Hospital de Infecciosos.

En el Laboratorio, aparte los análisis procedentes de las inspecciones del Cuerpo de Veterinaria o interesados por organismos oficiales y por particulares, se elaboran constantemente gran número de vacunas, no sólo anti-variólicas, sino también anti-diftéricas, antitifoideas, Calmette, y en la actualidad contra el tifus exantemático, siendo un Centro de trabajo constante y un colaborador, no sólo de las instituciones higiénicas del Estado y de la Provincia, sino también de los elementos técnicos particulares, constituyendo para ellos un lugar de estudio,. muy visitado y utilizado.

El Instituto Municipal de Higiene se desdobla en actividades de tipo prevencional, por medio de vigilancia e inspecciones sanitarias y formación de estadísticas, viniendo a ser un complemento del Laboratorio en esta materia; y en elemento activo de la Sanidad, con sus servicios de desinfección, desinsectación y desratización, practicados oficialmente o a petición de los particulares interesados.

Por último, el Hospital de Infecciosos, edificio modernísimo, reconstruído por haber sufrido importantes daños en la guerra, ha sido dotado de salas de aislamiento y de todos los adelantes modernos, en forma de hacerle perder el antiguo aspecto de hospital, para darle, no sólo por estas condiciones de edificación, sino también por su emplazamiento junto al mar, la alegría y la vistosidad de un sanatorio o clínica particular. Es capaz para 550 camas y es notable78. mente frecuente la hospitalización de personas pudientes, afectas de 
enfermedades infecciosas, única finalidad que cumple, por estar prohibida la hospitalización de las de otra índole.

Suple totalmente las instituciones de esta naturaleza de la Provincia, por ser único en ella.

2. Sanidad.-El Instituto de Asistencia Médica Domiciliaria, organizado a través de 10 Dispensarios y tres Casas de Socorro, atiende la beneficencia municipal con toda amplitud, pues además de los Dispensarios generales citados, funcionan tres Dispensarios de especialidades de oftalmología, otorinolaringología y venerología y urología.

Completan dichos servicios el Parque de Farmacia Municipal, para las atenciones de los servicios higiénicos y de los Hospitales que se examinan en el apartado de Beneficencia, y para las recetas del Instituto de Asistencia Médica Domiciliaria y sus Dispensarios, y asimismo la subvención de 350.000 pesetas anuales que el Ayuntamiento abona al Hospital Clínico por la atención de traumáticos, ya sea por accidentes del trabajo o por los de toda clase producidos en la vía pública, con lo que se cumple la doble finalidad de auxiliar a dicha organización estatal y de atender el servicio sin duplicidad y como se merece.

3. Cementerios.-Ni que decir tiene que los servicios de tal naturaleza se encuentran totalmente municipalizados, constituyendo unc de los más graves problemas que, como la depuración de aguas residuales a que se ha hecho referencia en el grupo de los de Vialidad, tiene planteados el Ayuntamiento para su urgente resolución.

El crecimiento. de la Ciudad llegó hasta su Cementerio, que hoy se llama Viejo, emplazado en la barriada que se denominaba entonces Pueblo Nuevo, junto al mar, y dejó dentro de las zonas urbanizadas los Cementerios de algunos de los pueblos agregados, con la consiguiente perturbación. En tal momento se resolvió el problema, construyéndose un Cementerio en la Montaña de Montjuich, frente al mar, es decir, dando espalda a la Ciudad, y' con una extensión de terreno que ha sido suficiente durante medio siglo. Ha tenido, no obstante, el gravísimo inconveniente de la dificultad de sus transportes interiores, por su enorme extensión y sus grandes pendientes, y el coste desmesurado de las construcciones, por tener que horadar unas veces la piedra viva de la montaña y otras que construirse taludes para el sostenimiento de tierras. Mas agotada prácticamente la extensión destinada a tal fin, se encuentra hoy la Ciudad en el grave trance de tener que buscar emplazamiento adecuado para un nuevo Cementerio; emplazamiento que, como es natural, no puede 
ser hecho dentro de los límites urbanos, sin que sea aconsejable llevarlo al extrarradio, pues por la topografía de Barcelona no puede hallarse en él superficie suficiente para tal fin.

Como se dice al hablar del emplazamiento del nuevo Matadero, hay que pensar también en que el emplazamiento del nuevo Cemen. terio debe buscarse fuera del término municipal y cerca, como es natural, de uná vía ferroviaria o con una buena comunicación por carretera.

Entre tanto el problema se va venciendo haciendo nuevas construcciones en el recinto de algunos de los Cementerios de los pueblos agregados, tales como los de Las Corts y San Andrés, cuyos emplazamientos en extrarradio permiten aún albergar unos miles de sepulturas.

Cuenta en conjunto el Ayuntamiento con nueve Cementerios, cun una extensión superficial total de 157.987,54 metros cuadrados, que da idea de la que precisará el nuevo emplazamiento.

\section{F) Los servicios de Beneficencia.}

La acción municipal en la materia se encuentra claramente clasificada en los dos aspectos de Asilos y Hospitales. Unos y otros exceden realmente, como hemos visto al examinar las instituciones del apartado anterior, a la competencia y a las necesidades estrictamente municipales. Pero así como decimos al principio que dado el crecimiento de la Ciudad, la urbanización debe ser prevista en relación a la de los pueblos contiguos, así en materia de higiene, tanto física como moral, es indudable que la gran Ciudad debe excederse, pues no ha de consentir que los males físicos de los pueblos vecinos puedan llegar a aquejarla a ella, ni ha de dejar que las miserias que la atracción de gran número de personas hecha constantemente por la Ciudad produce en gran parte de las atraídas, no encuentre en ella alivio.

1. Asilos.-El Asilo del Parque para ancianos tiene un carácter de preventorio más que de lugar definitivo, y su finalidad es albergar a los de uno y otro sexo en tanto encuentran acogida definitiva en los de fundación particular, muchas veces restringidos, o en los de carácter provincial. Su cabida de 510 plazas asegura sobradamente el servicio, tanto que su finalidad queda con frecuencia ampliamente rebasada por la permanencia durante años de muchos asilados.

80 El Asilo de Nuestra Señora del Puerto, construído después de 
la liberación, está destinado a la recogida de indigentes y a la ca rrección de los mendicantes profesionales. Su cabida de 300 plazas es suficiente para el internamiento de los que deben ser repatriados a sus pueblos de origen y para la corrección de los profesionales mediante el trabajo. Las mujeres en ocupaciones propias de sus habituales labores y los hombres en trabajos de campo y huerta, que a veces prestan en viveros próximos propiedad del Municipio.

Completa este servicio la antigua Colonia Puig, emplazada en la Rabasada, a mited de la ladera del Tibidabo, que, destinada antes a Colonia escolar de verano, ha sido habilitada ahora para albergar a los niños y niñas mendicantes menores de catorce años, para su corrección y devolución a sus padres, si los tienen o internamiento en Asilos definitivos. Esta Institución, juntamente con la Colonia Agrícola de Martorellas para chicos también medicantes de catorce a dieciocho años, situada a veinte kilómetros de la Ciudad, en finca también propiedad municipal, han asegurado completamente la recogida de mendigos, salvando así a la Ciudad de esa lacra física y social.

En construcción se encuentra además, en la calle de Valldoncella, dentro del casco antiguo de Barcelona, un albergue nocturno para facilitar cama a los indigentes, calculándose su capacidad en 250; lo que unido a los servicios de la Comisaría de Beneficencia instalada en su proximidad, donde se hace la desinfección y clasificación de todas estas personas. completan íntegramente el servicio, cubriendo las necesidades benéficas de la Ciudad.

2. Hospitales.-Cinco son las instituciones de esta naturaleza, de mayor o menor importancia, sostenidas íntegramente por el Presupuesto municipal.

En primer lugar figura el Nosocomio instituído, con la misma finalidad que algunos de los Asilos antes mencionados, como lugar de recogida y clasificación de los enfermos menesterosos para su ingreso en los Hospitales generales o de especialidades, en forma tal que en un tiempo se le denominó Preventorio, pues su finalidad no era otra que recoger el enfermo en tanto tuviera lugar su ingreso en la institución adecuada para su enfermedad. Tiene una capacidad de 80 camas, que aún cuando son suficientes, desgraciadamente suelen encontrarse casi totalmente ocupadas.

El Hospital de Incurables ha venido a cubrir una necesidad sentida en la Ciudad y en la Provincia, por encontrar ingentes dificultades para su hospitalización los enfermos de tal naturaleza; su nombre de Hospital de Nuestra Señora de la Esperanza confirma para 81 
ıos pobres en él acogidos la seguridad de un trato a su dilatada enfermedad, dejando por otra parte libres en los Hospitales generales camas que, ocupadas por enfermos de tal naturaleza, eran restadas a los agudos, que tal vez sucumbieran por no haber podido ser atendidos en su lugar. Actualmente ocupa un local arrendado con 125 camas, encontrándose en construcción un nuevo edificio, en la barriada denominada precisamente de la Salud, por su excelente emplazamiento, capaz para 600 camas y de un coste total de 3.750 .000 pesetas, que ha de asegurar cabida a todos los enfermos incurables.

El Instituto Psiquiátrico y el Neurológico, instalado uno en edificio propiedad del Municipio y el otro en local arrendado, son suficientes para atender al rápido internamiento de estos enfermos, con una cabida total de 230 camas, que cumplen con holgura el servicio municipal obligatorio de tal naturaleza. incluso con amplitud, pues corrientemente acuden también a dicha organización enfermos de pago, que en sus instalaciones modernas encuentran el confort adecuado a su posición social.

Completa el servicio la Casa de Maternología, que asiste a la mujer, más que en el momento del parto, durante su embarazo y en la lactancia de sus pequeños, sin perjuicio de internamiento y asistencia en su quirófano en los casos de urgencia o de gravedad. Sus 25 camas serían evidentemente insuficientes para la Ciudad de Barcelona si la Casa Provincial de Maternidad no asegurara con sus instalaciones ampliamente el servicio, por lo que, y dentro de la colaboración establecida y de las obligaciones que corresponden al Ayuntamiento, la Casa de Maternología va encaminada directamente, como se dice antes, a las dos atenciones de la mujer embarazada y del niño lactante, y no en el momento del parto.

\section{O N C L U S I O N}

De la exposición hecha en la primera parte de este artículo se deduce que la construcción de la Ciudad no pudo alcanzar un paso suficientemente acelerado para seguir el ritmo de crecimiento de la población. Al examinar en la segunda parte los servicios; a pesar de la forma somera en que ha sido hecha, se constata que algunos consiguieron alcanzar aquella marcha y que otros se encuentran retrasados. Al examinar el detalle de unos y otros, puede fácilmente comprobarse que los de mayor coste en su primer establecimiento son los que más atrasados quedaron. Ello confirma que las dificulta-

82 des del Ayuntamiento de Barcelona han sido todas de orden econó- 
mico, porque cuando el servicio ha podido establecer con un pequeño coste, se ha conseguido dotarlo y mantenerlo con el decoro preciso para la Ciudad, pero a medida que el coste de primer establecimiento es más importante, más difícil ha sido su montaje y dotación completa.

Esta consecuencia, que salta a la vista del examen hecho del crecimiento de la Ciudad y del desarrollo de sus servicios, deberá tener una plena y completa justificación en otro artículo que seguirá, dedicado a la Hacienda municipal del Ayuntamiento de Barcelona.

\section{ENRIQUE DE JANER}

Secretario interino

del Ayuntamiento de Barcelona. 